\title{
THE STATUS OF THE DIRBE INSTRUMENT ON THE COBE
}

\author{
M.G. HAUSER \\ NASA/Goddard Space Flight Center \\ T. KELSALL, H. MOSELEY, R. SILVERBERG, T.L. MURDOCK \\ General Research Cooperation, Danvers, Massachusetts \\ J.C. MATHER \\ NASA/Goddard Space Flight Center \\ G. SMOOT \\ University of California at Berkeley \\ R. WEISS \\ Massachusetts Institute of Technology \\ and \\ E.L. WRIGHT \\ University of California at Los Angeles
}

\begin{abstract}
The Diffuse Infrared-Background Experiment (DIRBE) on the Cosmic Background Explorer (COBE) satellite is a 10-band absolute photometer covering the wavelengths 1-300 microns using photovoltaic, photoconductive, and bolometric detectors. The input is via a $19-\mathrm{cm}$, off-axis, highly-baffled Gregorian telescope, with the detectors located at a pupil plane so they share the same field of view $(0.7 \times 0.7$ degrees $)$. The whole assembly is mounted inside a $1.4 \mathrm{~K}$ super-fluid, liquid-He dewar, which is shared with the Far Infrared Absolute Spectrometer (FIRAS) instrument. Each day half of the sky is surveyed, as the line-of-sight of the DIRBE is canted 30 degrees to the COBE spin axis. The whole sky is fully observed in 6 months, as the spin axis precesses at about 1 degree per day. At present each sky pixel has been observed at least once. The basic findings on the general brightness of the sky - Zodiacal light and galaxy - are provided, as well as a synopsis of the advantages and disadvantages associated with a space-borne observatory. The relationship of our experience and findings with respect to possible future missions and their scientific goals is presented.
\end{abstract}

Y. Kondo (ed.), Observatories in Earth Orbit and Beyond, 19.

(C)1990 Kluwer Academic Publishers. Printed in The Netherlands. 